\title{
Optimal Design of Survivable Mesh Networks Based on Line Switched WDM Self-Healing Rings
}

\author{
Andrea Fumagalli, Member, IEEE, Isabella Cerutti, Member, IEEE, and Marco Tacca, Member, IEEE
}

\begin{abstract}
Network survivability provided at the optical layer is a desirable feature in modern high-speed networks. For example, the wavelength division multiplexed (WDM) self-healing ring (or SHR/WDM) provides a simple and fast optically transparent protection mechanism against any single fault in the ring. Multiple self-healing rings may be deployed to design a survivable optical mesh network by superposing a set of rings on the arbitrary topology. However, the optimum design of such a network requires the joint solution of three subproblems: the ring cover of the arbitrary topology (the RC subproblem); the routing of the working lightpaths between end node pairs to carry the offered traffic demands (the WL subproblem); and the provisioning of the SHR/WDM spare wavelengths to protect every line that carries working lightpaths (the SW subproblem). The complexity of the problem is exacerbated when software and hardware requirements pose additional design constraints on the optimization process.
\end{abstract}

The paper presents an approach to optimizing the design of a network with arbitrary topology protected by multiple SHRs/WDM. Three design constraints are taken into account, namely, the maximum number of rings acceptable on the same line, the maximum number of rings acceptable at the same node, and the maximum ring size. The first objective is to minimize the total wavelength mileage (working and protection) required in the given topology to carry a set of traffic demands. The exact definition of the problem is given based on an integer linear programming (ILP) formulation that takes into account the design subproblems and constraints and assumes ubiquitous wavelength conversion availability. To circumvent the computational complexity of the exact problem formulation, a suboptimal solution is proposed based on an efficient pruning of the solution space. By jointly solving the three design subproblems, it is numerically demonstrated that the proposed optimization technique yields up to $12 \%$ reduction of the total wavelength mileage when compared to solutions obtained by sequentially and independently solving the subproblems. The second objective is to reduce the number of wavelength converters required in the solution produced by the ILP formulation. Two approaches are proposed in this case that trade the required wavelength mileage for the number of wavelength converters.

Manuscript received March 15, 2000; revised March 2, 2002; approved by IEEE/ACM TRANSACTIONS ON NETwORKING Editor K. Sivarajan. This work was supported in part by the U.S. Army Space and Strategic Defense Command under Contract DASG60-97-C-0050, by Alcatel, and by the National Science Foundation under Contract ANI-0082085. This paper was presented in part at the IEEE INFOCOM, New York, NY, 1999 and the IEEE IC3N, Boston, MA 1999.

A. Fumagalli is with the Optical Networking Advanced Research (OpNeAR) Laboratory, University of Texas at Dallas, Richardson, TX 75083-0688 USA (e-mail: andreaf@utdallas.edu).

I. Cerutti and M. Tacca were with Boston Communications Networks Inc., Waltham, MA 02154 USA. They are now with the Optical Networking Advanced Research (OpNeAR) Laboratory, University of Texas at Dallas, Richardson, TX 75083-0688 USA (e-mail: isabella_cerutti@ieee.org; mtacca@utdallas.edu).

Publisher Item Identifier 10.1109/TNET.2003.813039.
Index Terms-Self-healing ring, shared-line protection, survivability, wavelength converter.

\section{INTRODUCTION}

$A$ FTER more than a decade of research and extensive experimentation, wavelength division multiplexing (WDM) technology has become one of the key players in modern telecommunications networks. If, on one hand, WDM offers a viable solution to the increasing user's need for higher bandwidth, on the other hand the tremendous bandwidth made available by WDM is jeopardized by the occurrence of even a single fiber fault. When compared to conventional networks, such a fault is more catastrophic due to the unprecedented volume of traffic that can be accommodated in a single fiber, e.g., 160 wavelengths each one carrying $10 \mathrm{~Gb} / \mathrm{s}$ worth of traffic, and in a single cable, e.g., 100 fibers. A survivable WDM network design is therefore mandatory [1].

A network is considered survivable when it provides some ability to restore disrupted traffic demands due to a network component failure, such as a cable cut.

In addition to providing optically transparent paths of light, or lightpaths ${ }^{1}$ [2], [3], the optical (or WDM) layer ${ }^{2}$ may cost-effectively realize network functions that are typically provided by the electronic layers. Among these functions, protection switching is currently implemented at higher (electronic) layers, such as SONET/SDH, ATM, and IP [5], [6]. The recent availability of optical add and drop multiplexers (OADMs) and optical crossconnects (OXCs) [7] offers, however, a new dimension to make high-speed connections survivable. The advantages of providing a resilience mechanism at the optical layer include [8], [9]:

- fast and simple network fault recovery that higher layers may not be able to offer, e.g., upon failure of a single fiber a number of logical failures are detected at higher layers, thus unnecessarily complicating and delaying the recovery mechanism;

- prompt protection activation, i.e., higher layers must wait for alarms to propagate across lower (optical) layers;

- a protection technique that, due to the transparency of the wavelength routing mechanism [2], is independent of the protocols being used at the higher layers.

\footnotetext{
${ }^{1} \mathrm{~A}$ lightpath is a path of light between a source and a destination node, in which the wavelength may vary from one line to another, i.e., wavelength converters may be necessary at some intermediate nodes.

${ }^{2}$ Defined by the International Telecommunications Union (ITU) [4].
} 
A survivable WDM network can thus provide a reliable transmission medium, independently of the protocols and of the applications sharing that medium.

Among the available mechanisms that provide resilience to network failures at the optical layer, bidirectional shared-line WDM self-healing rings (SHRs/WDM) appear to be simple and practical [10]. Upon failure of a ring line, only the two nodes immediately adjacent to the faulted line need to perform rerouting of the interrupted traffic demands, by resorting to counter rotating spare wavelengths [5]. Signaling required to manage the fault is locally confined within the ring where the fault occurs. Therefore, a fast recovery action is possible with a recovery time proportional to the ring latency and size. (Notice that the designer can limit the maximum ring size to bound the recovery time as dictated by the higher layer applications. A similar feature is not available in end-to-end shared-path protection mechanisms [11] in which the recovery time is proportional to the connection end-to-end propagation latency.) Another appealing feature of the shared-line SHR/WDM protection mechanism is the capability of sharing the spare wavelengths provisioned to protect multiple lines and thus of ensuring a cost effective protection solution against any single line fault in the ring. For example, it can be numerically demonstrated that in an arbitrary topology (in this paper, also referred to as mesh) the network capacity required by superposing multiple SHRs/WDM, called multi-SHR/WDM design, is approximatively equivalent to the capacity required by dedicated end-to-end path protection (Section V). Multi-SHR/WDM design can handle multiple faults too, as long as the faults take place on lines covered (protected) by distinct rings. Last but not least, the rerouting mechanism in SHRs/WDM has been successfully demonstrated in a number of field trials [10].

Although several resource provisioning problems have been addressed in stand-alone rings [5], [11]-[14], the problem of designing an arbitrary topology by superposing multiple SHRs/WDM has been marginally studied. In [6] and [15], a hierarchy of interconnected rings is proposed to design multi-ring SONET transport networks. In [16], SHRs with shared-line protection are considered to protect the offered traffic in a mesh network. Traffic routing is performed using the shortest path, in conjunction with a load balancing algorithm applied to choose among equal length routes. The ring cover is determined after the routing is completed. In [17], a SONET ring-like protection mechanism is used in the optical layer. Resource optimization in the network is achieved sequentially: first, working connections are routed, and then, spare resources are provisioned in each ring. In [18], the proposed design process consists of three steps that are handled sequentially: find all possible ring candidates in the given topology, select a subset of actual rings for the cover, and distribute the traffic demands over the rings of the cover. The ring selection is based on the assumption that every node pair exchanging traffic is connected by a single ring.

None of the above works takes into account the potential design constraints that may originate due to software and hardware complexity and may limit the number and size of the rings in the cover. Solving the problem of optimally designing an arbitrarily topology based on SHRs/WDM under these design constraints is not, in general, a trivial task.

This paper defines the problem of optimally providing SHR/WDM protection in an arbitrary topology, taking into account the following design constraints:

1) the maximum number of SHRs/WDM sharing a line is bounded;

2) the maximum number of SHRs/WDM sharing a node is bounded;

3) the SHR/WDM maximum size (number of nodes) is bounded.

The considered problem, referred to as the WDM self-healing rings with line protection (or WRL) problem, consists of three subproblems:

- WL subproblem: for every traffic demand, route the working lightpath(s);

- RC subproblem: for every line carrying at least one working lightpath, identify the ring(s) covering the line and protecting the traffic;

- SW subproblem: for every ring in the cover, provision the spare wavelengths that are necessary to protect the working lightpaths.

The three WRL subproblems are related to one another and must be jointly solved in order to yield best design.

In solving the WRL problem, two objectives are considered in this paper.

The first objective is to minimize the total (working and protection) wavelength mileage required in a given network topology to support a given set of traffic demands without violating the design constraints. The wavelength mileage is minimized here assuming ubiquitous wavelength conversion availability in the network. As shown, the search for the optimum solution of the WRL problem, based on the integer linear programming (ILP) formulation presented in Section III, is computationally intensive already for networks with few tens of nodes. Alternative optimization approaches must be therefore investigated, while still striving for a joint solution of the three subproblems. An approach based on the reduction of the search space is thus proposed in Section III-C. Pruning is achieved by relying upon a preselected subset of ring candidates and a preselected subset of path candidates in the solution of the RC and WL subproblem, respectively. Numerical results show that, while increasing the network wavelength mileage by only few percents with respect to the optimum solution, the suboptimal ILP approach significantly reduces the necessary computational time. Comparison with other approaches that sequentially and independently deal with the three WRL subproblems reveals that the joint solution of the WRL subproblems yields up to $12 \%$ reduction of the network total wavelength mileage.

The second objective is to limit the use of wavelength converters by enforcing the wavelength continuity constraint within each ring and making use of wavelength conversion only when a lightpath is crossconnected from one ring to another. The advantage of this approach is to require converter-free OADMs, and wavelength conversion only at the OXCs where other functions, such as signal regeneration, are expected to 
take place. Two heuristics are presented whose aim is to limit the number of converters by modifying the solution produced by ILP formulation of Section III, i.e., rings and working lightpaths computed by the ILP are taken into account by the heuristics. The first heuristic (re)maps each working lightpath onto the minimum number of required rings, thus minimizing the number of converters required by each lightpath. Traffic balancing with wavelength continuity [19] is then performed within each ring to minimize the wavelength mileage. The second heuristic (re)maps the working lightpaths onto the rings in order to reduce the total number of times lightpaths are crossconnected, while maintaining constant the wavelength mileage found by the ILP. Wavelength continuity is then enforced within each ring, which may marginally increase the original wavelength mileage found by the ILP.

With the proposed suboptimal ILP and heuristic solutions, it is possible to find the desired tradeoff between the required wavelength mileage and number of converters. More precisely, priority shifts from wavelength mileage to number of converters in the following order: the ILP solution, the second heuristic, and the first heuristic.

\section{Mesh NetWork With WDM Bidirectional SHARED-LINE RING PROTECTION}

This section describes a WDM network with arbitrary topology ${ }^{3}$ in which protection is achieved by means of bidirectional shared-line SHRs/WDM [20].

Working lightpaths are established between node pairs to support traffic demands. A lightpath can rely upon multiple rings if necessary. Optical crossconnect capabilities are required only at nodes where working lightpaths hop from one protection ring to another (Fig. 1). The other nodes need only optical add and drop multiplexing capabilities. Protection for each lightpath is provisioned by indicating the rings protecting each line of the lightpaths. If multiple rings cover the same line, a lightpath on that line must be assigned to one ring only for protection purposes. For simplified hardware and control, it is assumed that multiple rings cannot share fibers, nor spare wavelengths.

The same protection mechanism of a stand-alone SHR/WDM is used in the presence of multiple rings covering the mesh network. In case of a line fault, the two nodes immediately adjacent to the fault stop transmitting on the faulted line and reroute the interrupted working lightpaths along the provisioned counter rotating spare wavelengths. In each ring, the number of provisioned spare wavelengths with clockwise (counterclockwise) transmission is constant across the ring lines, and equal to

$$
\max _{i \in \text { ring }}\left(\# l p_{i}\right)
$$

where $\# l p_{i}$ is the number of counterclockwise (clockwise) working lightpaths on line $i$ that are assigned to the ring. Notice that in a SHR/WDM, the number of provisioned clockwise spare wavelengths does not necessarily equal the number of provisioned counterclockwise spare wavelengths (Fig. 1).

\footnotetext{
${ }^{3}$ The only necessary hypothesis is that the graph representing the topology is two-connected. A graph that is not two connected does not allow protection of some lines.
}

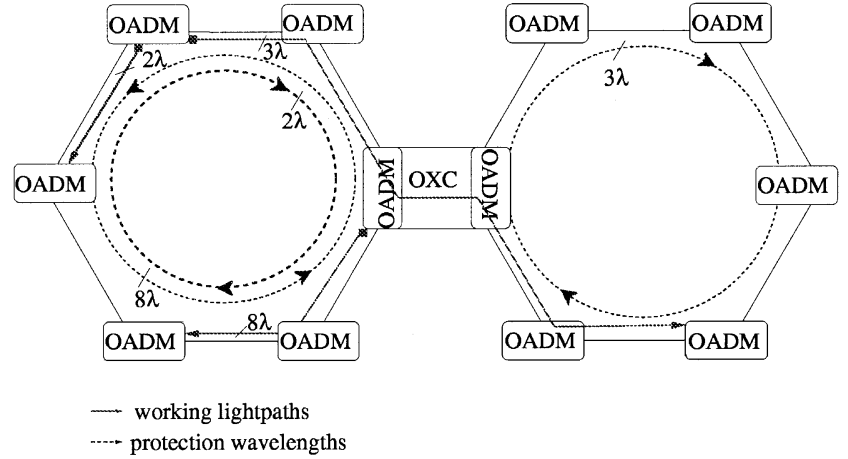

Fig. 1. Shared-line protection scheme.

Cost-effective design of the SHR/WDM-based mesh network depends on a number of factors, including the network topology, the traffic matrix, the ring cover, the design constraints posed on the ring cover, and the objective function.

In the remaining sections, two distinct scenarios are considered: wavelength conversion is available in both OADMs and $\mathrm{OXCs}$ - referred to as the ubiquitous wavelength conversion availability case-and wavelength conversion is available only in the OXCs, i.e., the wavelength of a lightpath can be changed only when crossconnecting from one ring to another (Fig. 1)-referred to as the limited wavelength conversion availability case.

\section{UbiQuitous WAVELENGTH CONVERSION AVAILABILITY CASE: ILP FORMULATION OF THE WRL PROBLEM}

The WRL problem, or WDM self-healing rings with line protection problem, is first solved with the objective of minimizing the total (working and protection) wavelength mileage required in an arbitrary topology to carry a given set of traffic demands without violating a given set of design constraints. An exact ILP formulation of the WRL problem is presented under the following assumptions:

1) The line capacity (number of wavelengths) is not bounded;

2) Every traffic demand (lightpath) must be protected against any possible single line failure;

3) Shared-line SHR/WDM protection mechanism is used;

4) Distinct rings superposed on the same line cannot share the same spare wavelength, i.e., a spare wavelength can be provisioned to one ring only (fibers may be shared in the proposed formulation);

5) Wavelength conversion is available in both OADMs and OXCs.

It is also assumed that the given arbitrary topology and traffic matrix are such that a feasible solution of the WRL problem exists [21] in the absence of design constraints.

\section{A. Definitions}

\section{Input parameters:}

- $G(V, E, W)$ : a directed graph representing the arbitrary topology, where $V$ is the set of nodes, $E$ is the set of weighted links (bidirectional lines are replaced with a pair 
of directed links), and $W$ is the weight function. For each link $(i, j) \in E, W(i, j)$ is the length in miles of line $(i, j)$; - $N=|V|, L=|E|$

- $\Lambda$ : the traffic matrix whose entries are defined as the number of lightpaths required between any node pair, e.g., $\Lambda_{s d}=2$ implies that two lightpaths from node $s$ to node $d$ must be set up;

- $R^{*}$ : set that contains all the rings that can be drawn on graph $G(V, E, W)$. Rings in this set are ordered from 1 to $r_{\text {max }}=\left|R^{*}\right|$

- $P_{s d}^{*}$ : set that contains the set of paths between node pair $(s, d)$. One set is defined for each node pair. Paths in $P_{s d}^{*}$ are ordered from 1 to $p_{\max }^{s d}=\left|P_{s d}^{*}\right|$;

- binary $k_{m n}^{s d j}: 1$ if link $(m, n) \in E$ belongs to the $j$ th candidate path between node pair $(s, d), 0$ otherwise;

- binary $a_{m n}^{r}: 1$ if link $(m, n) \in E$ belongs to ring $r, 0$ otherwise;

- binary $\overrightarrow{c_{m n}^{r}}\left(c_{m n}^{\overleftarrow{r}}\right): 1$ if traffic on line $(m, n) \in E$ requires clockwise (counterclockwise) protection on ring $r, 0$ otherwise;

- real $w_{s d}^{j}$ : length in miles of the $j$ th path candidate for node pair $(s, d)$

- real $l^{r}$ : length in miles of the $r$ th ring candidate;

- integer $S_{r}$ : number of nodes that belong to the $r$ th ring candidate.

\section{Constants:}

- $N_{l}$ : maximum number of rings allowed on a line;

- $N_{v}$ : maximum number of rings allowed per node;

- $N_{r}$ : maximum number of nodes/lines a ring can have;

- $T_{\mathrm{Max}}$ : a constant greater than the number of working lightpaths on any link of the graph;

- $C_{\mathrm{Max}}$ : a constant greater than the number of provisioned spare wavelengths on any link of the graph.

\section{Variables:}

- integer $t_{s d}^{n}$ : number of working lightpaths from node $s$ to node $d$ routed along the $n$-th path candidate $\in P_{s d}^{*}$;

- binary $\delta^{r}: 1$ if ring $r$ is selected to cover the network, 0 otherwise;

- integer $c_{1}^{r}\left(c_{2}^{r}\right)$ : number of provisioned spare wavelengths in ring $r$ with the clockwise (counterclockwise) direction.

\section{B. Linear Problem}

The objective of the ILP formulation is to minimize the total wavelength mileage, the sum of the total working lightpath mileage and the total provisioned spare wavelength mileage, given sets $R^{*}$ and $P_{s d}^{*}$.

\section{Objective function}

$$
\min \left[\left(\sum_{s d} \sum_{j \in P_{s d}^{*}} w_{s d}^{j} \cdot t_{s d}^{j}\right)+\left(\sum_{r} l^{r} \cdot\left(c_{1}^{r}+c_{2}^{r}\right)\right)\right]
$$

\section{Subject to:}

WL subproblem:

$$
\sum_{j \in P_{s d}^{*}} t_{s d}^{j}=\Lambda_{s d} \quad \forall s, \quad d \in V, \quad s \neq d .
$$

This constraint ensures that all traffic demands are fulfilled.

\section{RC and SW subproblems:}

$$
\begin{aligned}
& T_{\text {Max }} \cdot \sum_{r} a_{m n}^{r} \cdot \delta^{r} \geq \sum_{s d} \sum_{j \in P_{s d}^{*}} k_{m n}^{s d j} \cdot t_{s d}^{j} \quad \forall(m, n) \in E \\
& c_{1}^{r}+c_{2}^{r} \leq C_{\mathrm{Max}} \cdot \delta^{r} \quad \forall r \in R^{*} \\
& \sum_{r}\left(c_{m n}^{r} \cdot c_{1}^{r}+c_{m n}^{r} \cdot c_{2}^{r}\right) \\
& \quad \geq \sum_{s d} \sum_{j \in P_{s d}^{*}} k_{m n}^{s d j} \cdot t_{s d}^{j} \quad \forall(m, n) \in E \\
& \sum_{r} a_{m n}^{r} \cdot \delta^{r} \leq N_{l} \quad \forall(m, n) \in E \\
& \sum_{r} \sum_{n} a_{i n}^{r} \cdot \delta^{r} \leq 2 \cdot N_{v} \quad \forall i \in V \\
& S_{r} \cdot \delta^{r} \leq N_{r} \quad \forall r \in R^{*} .
\end{aligned}
$$

Constraint (2) ensures that any line carrying at least one working lightpath is covered (protected) by at least one ring. Constraint (3) ensures that spare wavelengths are provisioned only in rings selected to cover the network. Constraint (4) ensures that all working lightpaths passing through line $(m, n) \in E$ are protected by provisioning the necessary spare wavelengths in the ring(s) that are selected to cover the line. Constraints (5), (6), and (7) ensure that the selected rings satisfy the design constraints on the maximum number of rings covering a line, the maximum number of rings covering a node, and the maximum number of nodes/lines a ring can have.

\section{Integrality constraints:}

$$
\begin{aligned}
& \text { int } t_{s d}^{j} \quad \forall s, d \in V \quad \forall j \in P_{s d}^{*} \\
& \text { binary } \delta^{r} \quad \forall r \in R^{*} \\
& \text { int } c_{1}^{r} \quad \forall r \in R^{*} \\
& \text { int } c_{2}^{r} \quad \forall r \in R^{*} .
\end{aligned}
$$

The WRL integer linear formulation has a number of variables that grows as $O\left(\sum_{s d} p_{\max }^{s d}+r_{\max }\right)$ and a number of constraints that grows as $O\left(L+N^{2}+r_{\max }\right)$.

\section{Pruning the Search Space of the WRL Problem}

The complexity of the exact linear formulation of the WRL problem, described in the previous section, heavily limits the size of the networks that can be designed optimally. The reason is the exponential growth of both the number of rings [22] and the number of possible paths as a function of the number of nodes $N$. A simplified suboptimal approach to solving the WRL problem is therefore proposed in this section to provide a practical way to design current existing networks, such as the European network that consists of $N=19$ nodes. The suboptimal approach, referred to as the SO-WRL, trades complexity for optimality by intelligently pruning the solution search space.

Pruning in the SO-WRL solution is based on the following observations. Let $R \subset R^{*}$ be a subset of predetermined ring candidates that is likely to contain most of the rings found by the optimum solution. Let $P_{s d} \subset P_{s d}^{*}$ be a subset of predetermined path candidates for any given source-destination pair $(s, d)$ that is likely to contain most of the working lightpaths found in the 
optimum solution. By restricting the set of predetermined ring candidates and path candidates to take into account only the rings in $R$ and the working lightpaths in $P_{s d}$, the complexity of the WRL problem can be greatly reduced. However, the selection of both $R$ and $P_{s d}$ is a critical step since some of the rings and paths that are actually part of the optimum solution may be mistakenly pruned out. Two algorithms are proposed to accomplish this task:

- Shortest Ring (SR) algorithm;

- Minimum Ring Distance Path (MRDP) algorithm.

1) SR Algorithm: As the number of possible rings (cycles) in a graph grows exponentially with the graph size, the ring pruning must reduce drastically the number of ring candidates to be included in set $R$. At the same time, since the selection of the rings that will cover the network must be performed jointly with the routing of the working lightpaths and the provisioning of the spare wavelengths, it is important that the number of ring candidates is larger than the number of rings that will be chosen to cover the network. This fact will provide the WRL linear formulation the necessary dynamics to still select the set of optimal rings from the set of ring candidates.

The algorithm designed to perform the ring pruning is based on the observation that the minimum total wavelength mileage for a single traffic demand is achieved by selecting the shortest ring that connects both source and destination [18]. Rings in set $R$ are thus chosen to be the shortest rings between each node pairs that do not exceed the allowed maximum size. A detailed description of the algorithm follows.

Define the ring weight as the summation of the weights of the ring lines. Define $S_{r}$ as the number of nodes forming ring $r$, i.e., the ring size. Given a node pair, $(s, d) \in V$, the shortest ring with respect to that pair, $r_{s d}$, is defined as the ring connecting both nodes with the minimum weight. Define the set of shortest rings in graph $G$ whose size does not exceed the allowed maximum ring size, $N_{r}$, as $R_{s}=\left\{r_{s d}, \forall(s, d) \in V \mid S_{r_{s d}} \leq N_{r}\right\}$.

Setting $R=R_{s}$ seems to be a reasonable approach. However, in a sparse graph, it may happen that, due to the imposed maximum ring size, set $R$ does not cover all the nodes and/or lines in the graph, thus potentially leaving some working lightpaths unprotected. Under this circumstance, the WRL problem does not have a feasible solution unless additional rings that violate the size constraint $N_{r}$ are added to set $R$. In this case, in order to have each node and each line in the graph covered by at least one ring, set $R$ is augmented by first adding the minimum weight rings that complete the node covering. After this step, some lines (possibly with higher weight) may still not be covered by any ring in $R$. If so, set $R$ is further augmented by adding the minimum weight rings that complete the covering of all lines.

A formal description of the SR algorithm is given next.

$$
\begin{aligned}
& \text { begin } \\
& R=\emptyset \\
& N=|V| \text {, number of nodes in the network } \\
& N_{r \max }=\text { initial maximum size of the ring } \\
& N_{r \max }=N_{r} \\
& \text { for (each node pair) }
\end{aligned}
$$

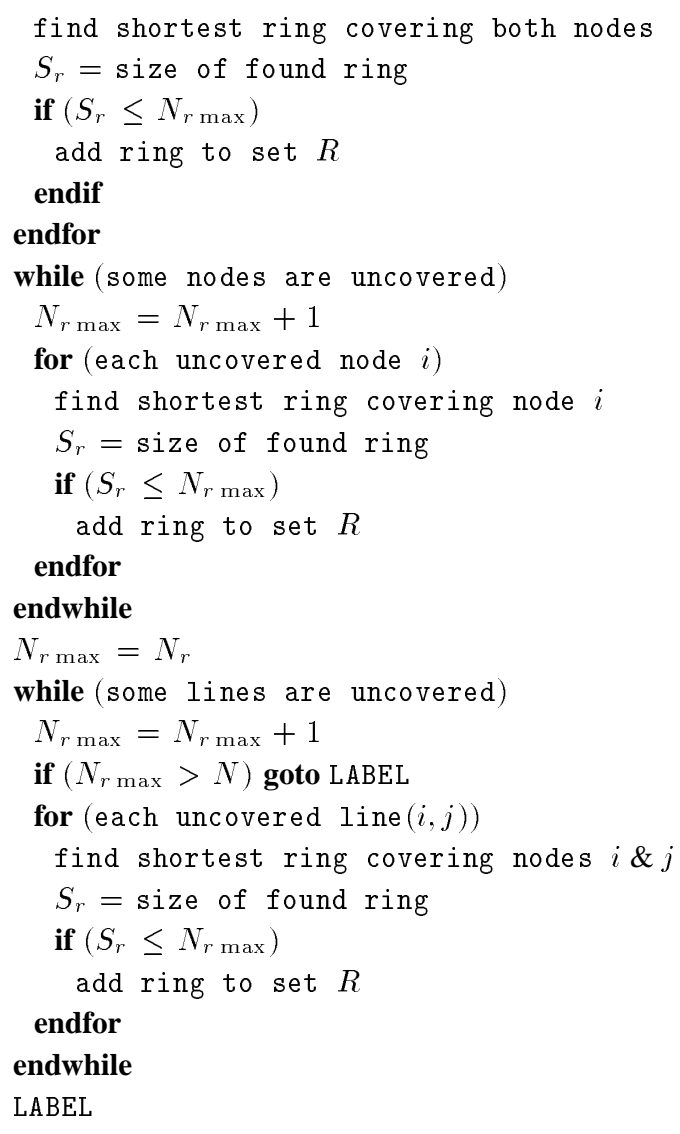

2) MRDP Algorithm: The pruning of the paths to obtain set $P_{s d}$, for every node pair $(s, d) \in V$, is based on the conjecture that a candidate path must rely upon the minimum number of rings. This conjecture is supported by two observations. First, since a crossconnect is necessary to switch a working lightpath between two adjacent rings, the candidate path minimizes the required number of crossconnect ports (Section II). This choice tends to minimize the overall number of crossconnect ports in the network. Second, although the path candidate may not necessarily coincide with the shortest path, it tends to rely on large portions of the ring. As a result, a balanced distribution of the working lightpaths is easier to achieve within each ring.

Set $P_{s d}$ is constructed in two steps. Let $G^{\prime}\left(V^{\prime}, E^{\prime}, W^{\prime}\right)$ be an auxiliary graph where $V^{\prime}$ is the set of nodes and contains one node for each ring in $R, E^{\prime}$ is the set of links and contains link $(i, j)$, if ring $i$ and ring $j$ share at least one vertex in $G$, and $W^{\prime}$ is the weight function. Fig. 3 shows the auxiliary graph $G^{\prime}$ which is built from the graph $G$ shown in Fig. 2. The set of shortest paths, in the number of hops, between each node pair is derived on the auxiliary graph. The nodes along a shortest path in $G^{\prime}$ represent the sequence of rings that are crossed by a number of alternative paths in $G$ from $s$ to $d$. More specifically, each ring (vertex) that belongs to a shortest path in $G^{\prime}$ identifies two alternative paths in $G$, one per direction of propagation in the ring. Finally, for each identified sequence of rings in $G^{\prime}$, all possible alternative paths in $G$ from $s$ to $d$, that rely upon the rings in the sequence, are included in $P_{s d}$. Fig. 3 shows an example of a ring sequence in the auxiliary graph. Fig. 2 shows the corresponding paths in 


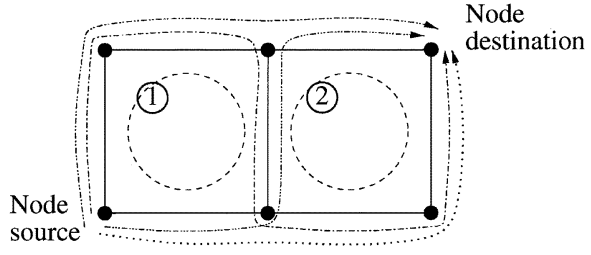

Fig. 2. Alternative paths.

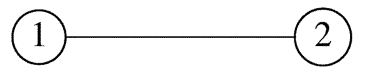

Fig. 3. Auxiliary graph.

$G$ identified by the ring sequence. The number of paths in $P_{s d}$ after this first step can be fairly large and can be shown to be at least $2^{p}$ for each ring sequence found, where $p$ is the number of rings in the sequence.

In the second step of the MRDP algorithm, the size of set $P_{s d}$ is further reduced to $k$ paths, where $k$ is a varying parameter that can be used to control the complexity of the ILP formulation. The paths in $P_{s d}$ are sorted by increasing number of nodes in $G$, and ties are broken by increasing path mileage. Only the first $k$ paths are kept in set $P_{s d}$; the others are dropped. The above choices are motivated by the observation that paths remaining in $P_{s d}$ cross the minimum number of add/drop multiplexers and at the same time have the minimal end-to-end power loss.

\section{LIMITED WAVELENGTH CONVERSION AVAILABILITy CASE}

This section addresses the scenario in which wavelength converters are present only in OXC nodes. The objective is to keep the number of required wavelength converters as minimal as possible. Since a wavelength converter may be needed every time a lightpath is bridged from one ring to another, the number of converters is bounded by the number of times a lightpath is crossconnected from one ring to another. This is equivalent to minimizing the number of rings that are required to protect a lightpath, which is the scope of this section.

Recall that the ring cover obtained as the solution of the RC subproblem may have up to $N_{l}$ rings covering the same line. Let such a line be defined as multi-covered line. Since spare resources cannot be shared among rings, any spare wavelength and fiber on a multi-covered line must be assigned to one ring only. Similarly, the portion of a working lightpath on a multicovered line must be protected by one ring only. The above assumptions are necessary to guarantee that only one protection mechanism for each working lightpath is triggered in case of a faulty multi-covered line. Assigning the portion of a working lightpath (that relies upon a multi-covered line) to a ring of the cover is defined as the ring assignment problem. It is important to notice that multiple solutions exist for the ring assignment problem, and that the chosen solution depends on the cost function defined by the designer.

An example is presented that illustrates the ring assignment problem using the network depicted in Fig. 4. Three lightpaths are shown $(l 1, l 2$, and $l 3)$ with three rings $(R 1, R 2$, and $R 3)$ covering the mesh. Assume that each ring is designed to protect at most one lightpath (or lightpath portion) on each multi-covered line, i.e., the set of spare wavelengths (and wavelength mileage)

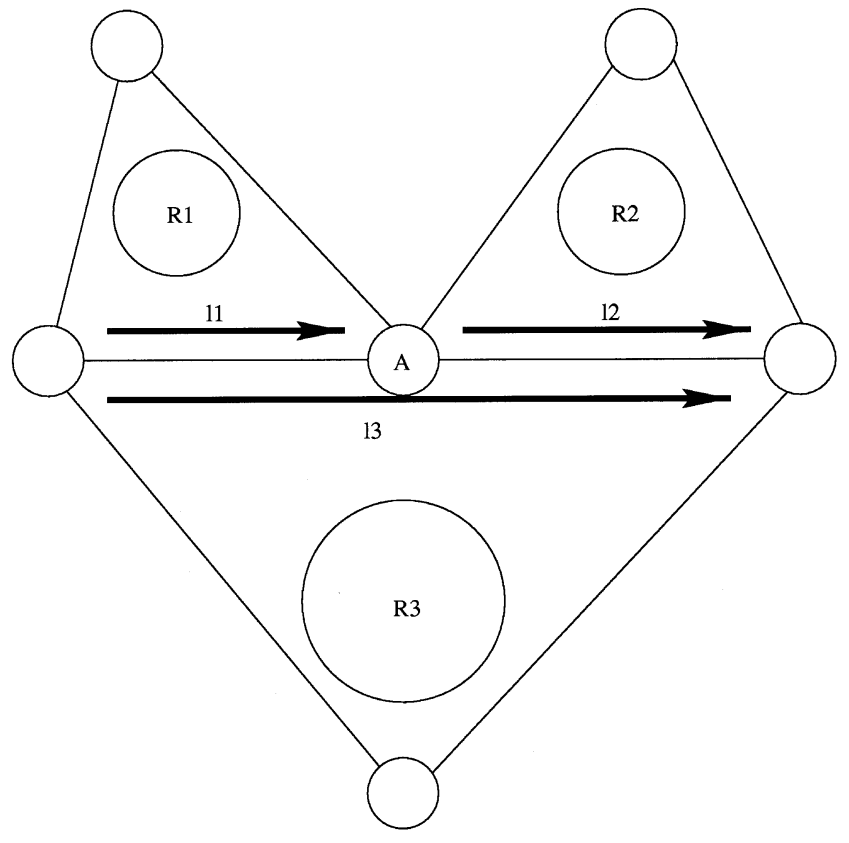

Fig. 4. Ring assignment.

in the rings is given and fixed. For this network, the ring assignment problem has two solutions. If $l 1, l 2$, and $l 3$ are protected by $R 1, R 2$, and $R 3$, respectively, no wavelength converters are necessary as each lightpath is protected by one ring only. However, if lightpaths $l 1$ and $l 2$ are protected by ring $R 3$, and lightpath $l 3$ is protected using both ring $R 1$ and ring $R 2$, one wavelength converter may be required at node $A$ to crossconnect $l 3$ from $R 1$ to $R 2$. Although the problem in Fig. 4 has a trivial solution due to the simplicity of the network and the limited number of lightpaths and rings, generally speaking, the optimal solution of the ring assignment problem can be rather complex. In addition, in many cases the two cost functions, i.e., wavelength mileage and number of converters, have conflicting objectives.

Two heuristic approaches are proposed to solve the ring assignment problem. The first heuristic gives priority to the objective of minimizing the number of wavelength converters. The second heuristic gives priority to the objective of minimizing the wavelength mileage.

As an initial solution, both heuristics use the set $R_{o}$, i.e., the set of rings (RC subproblem), and the set $P_{o}$, i.e., the set of routes for the working lightpaths (WL subproblem), obtained by solving the ILP formulation of the WRL problem.

\section{A. Heuristic 1: Minimize the Number of Wavelength Converters First}

The first heuristic is based on the following two-step approach.

Step 1: Minimize the number of rings assigned to each working lightpath given both the ring cover $R_{o}$ and the set of working lightpaths $P_{o}$. For each lightpath, the ring assignment problem is solved by selecting the ring sequence with the smallest number of rings. The sequence is found by applying a shortest path algorithm [23] to the auxiliary graph built as described in the procedure in Section III-C2, using only the rings in the ring cover $R_{o}$. 


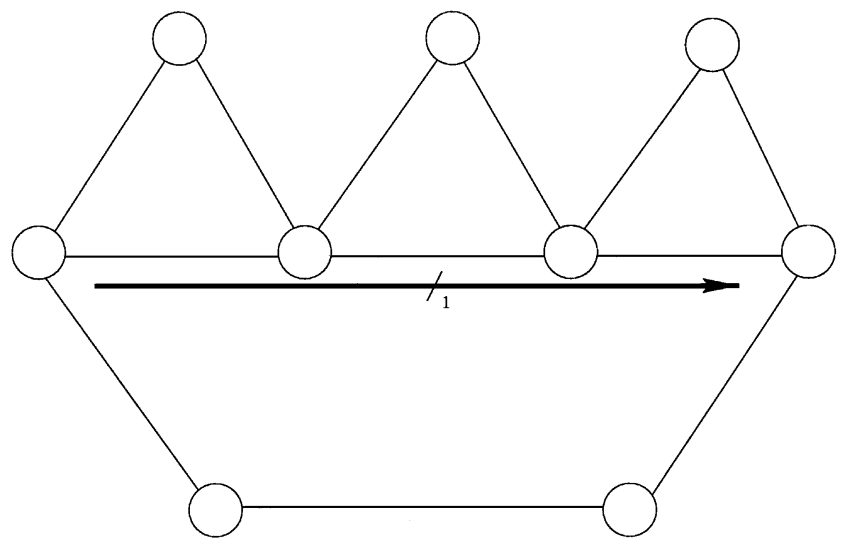

Fig. 5. $R D=3, r d=1 \longrightarrow(R D-r d)=2$.

The complexity of this step is $N^{2}\left|P_{o}\right|$, where $\left|P_{o}\right|$ is the cardinality of set $P_{o}$.

Step 2: Minimize the wavelength mileage of each ring without changing the working lightpath-ring assignments found in Step 1. Based on the result of Step 1, the capacity of each ring to ensure $100 \%$ protection against any single line fault is determined. To minimize the required wavelength mileage in each ring, the load balancing and wavelength assignment algorithm presented in [19] is used. ${ }^{4}$ By minimizing the wavelength mileage in each ring, the total wavelength mileage in the entire network is minimized. Notice that in order to balance the traffic some of the portions of the working lightpaths may be rerouted within a ring. Thus, the original given set of working lightpaths $P_{o}$ may be changed.

\section{B. Heuristic 2: Minimize the Wavelength mileage First}

The second heuristic is based on the following two-step approach.

Step 1: Minimize the number of rings assigned to each working lightpath, given the ring cover $R_{o}$, the number of provisioned spare wavelengths for each ring, and the set of working lightpaths $P_{o}$.

For each lightpath, two integer values are derived:

- $r d$ : the smallest number of rings required to protect the entire lightpath;

- $R D$ : the largest number of rings required to protect the entire lightpath (Fig. 5).

Subsequently, the lightpaths are sorted according to the nondecreasing difference $(R D-r d)$.

Considering one lightpath at a time in the order given by the sorting, the ring assignment for each lightpath is obtained by selecting the ring sequence with the minimum number of rings that still have available protection wavelengths. Protection wavelengths that are assigned to a lightpath will be no longer available for other lightpaths. Gradually, the protection wavelengths of all rings will be assigned to the lightpaths. During this step, ubiquitous wavelength conversion availability is still assumed, therefore the initial number of provisioned spare wavelengths is not increased.

\footnotetext{
${ }^{4}$ The assumption of wavelength conversion availability at OXCs and the assumption of no sharing of fibers among different rings make it possible to balance the load and to assign wavelengths on each ring independently.
}

The algorithm rationale is that lightpaths that carry more wavelengths and whose ring assignment may significantly affect the number of wavelength converters are considered first, i.e., when a large number of protection wavelengths are still available. In this way, a better-than-average selection is done on these critical lightpaths.

The complexity of this step is twice the complexity of Step 1 of the algorithm presented in Section IV-A. The shortest path algorithm must be run twice in order to first evaluate $r d$ and then to assign the set of protecting rings to each lightpath.

Step 2: Minimize the wavelength mileage of each ring without changing the working lightpath-ring assignments found in Step 1. This step is the same as Step 2 of the algorithm described in Section IV-A.

\section{NUMERICAL RESUltS}

The objectives of this section are:

- to assess the performance gap between the optimum and the SO-WRL solution;

- to compare the performance of the proposed joint optimization of the three WRL subproblems against the performance of other approaches in which the subproblems are solved sequentially;

- to evaluate both the wavelength mileage and number of wavelength converters obtained by the two heuristics proposed for the limited wavelength conversion availability case.

For demonstration purposes, two network topologies are considered: a seven-node benchmark network in which the optimum solution can be always found by the solver, and the European network that is conventionally used to evaluate scalability and complexity of the proposed design approaches. Numerical results are obtained by either solving the integer linear formulation using the lp_solve package [24], or running the heuristic custom software modules on a Linux PC computer.

\section{A. Seven-Node Benchmark Network}

A benchmark network is used to compare the results obtained using the WRL ILP formulation-the optimum solution and the SO-WRL approach — and some approaches that sequentially solve the three WRL subproblems.

Fig. 6 shows the benchmark network that consists of $N=7$ nodes and $L=22$ unidirectional lines. The weight (length in miles) of the lines is shown in the figure. A uniform traffic pattern of ten connections (lightpath demands) for any source-destination pair is assumed. The design constraints are the following.

- $N_{l}=2$ : maximum number of rings covering the same line.

- $N_{v}=4$ : maximum number of rings covering the same node.

- $N_{r}=7$ : maximum number of nodes a ring can have.

All results presented in Table I are optimal solutions of the corresponding problem formulation. In these experiments, the MRDP algorithm is run considering all possible candidate paths $(k \rightarrow \infty)$. 
TABLE I

BENCHMARK NETWORK: VARIOUS SOLUTIONS OF THE WRL PROBLEM.

\begin{tabular}{c|r|r|r|r|r|r|r}
\hline Sol \# & $\begin{array}{r}\text { total } \\
\lambda \text { miles }\end{array}$ & $\begin{array}{r}\text { working } \\
\lambda \text { miles }\end{array}$ & $\begin{array}{r}\text { protection } \\
\lambda \text { miles }\end{array}$ & rings & paths & $\begin{array}{r}\text { CPU } \\
\text { time }\end{array}$ & \\
\hline 1 & 15880 & 7940 & 7940 & all rings & all & 2 min & $0 \%$ \\
\hline 2 & 16080 & 8040 & 8040 & SR & MRDP & $5 s$ & $1.26 \%$ \\
\hline 3a & 17880 & 7620 & 10260 & SR & SP & $5 s$ & $12.59 \%$ \\
\hline 3b & 17160 & 7880 & 9280 & cover on all rings & MRDP on all & $0.97 s$ & $8.06 \%$ \\
\hline 3c & 17040 & 7680 & 9360 & cover on SR & MRDP on SR & $0.78 s$ & $7.3 \%$ \\
\hline
\end{tabular}

Table I shows the total wavelength mileage, the sum of the working miles and protection miles, for a number of solutions of the WRL problem in the benchmark network. The fifth and sixth columns show, respectively, the set of ring and path candidates used to obtain the solution. The seventh column shows the CPU time required by the $l p \_$solve package to find the optimal solution. The rightmost column shows the total wavelength mileage penalty percentage of the various solutions with respect to the optimum solution.

Solution 1 is the optimum solution and takes into consideration all possible ring and path candidates of the network. Solution 2 shows the results obtained using the SO-WRL approach. While losing optimality, the total mileage in solution 2 is only marginally increased (less than $2 \%$ ), while the CPU time is considerably reduced from the 2 minutes of solution 1 to $5 \mathrm{~s}$. Solutions $3 \mathrm{a}, 3 \mathrm{~b}$, and $3 \mathrm{c}$ are examples of sequential approaches. Solution 3a shows the results obtained using the shortest path (SP) as the only path candidate in $P_{s d}$. As expected, solution 3a yields the minimum working mileage. However, such routing does not permit an efficient sharing of the spare wavelengths to protect the working lightpaths, as demonstrated by the $12 \%$ penalty of the total wavelength mileage with respect to solution 1 . Solutions $3 \mathrm{~b}$ and $3 \mathrm{c}$ are obtained by first selecting the ring cover, then jointly deriving the routing of the working lightpaths and the provisioning of the spare wavelengths. The ring cover is obtained by minimizing the sum of the perimeter of the rings selected to obtain a full cover that is irrespective of the traffic matrix. To determine the cover, solution $3 \mathrm{~b}$ considers all rings as candidates, and solution $3 \mathrm{c}$ considers only the rings generated by the SR algorithm. Solution $3 \mathrm{~b}$ is slightly worse than solution $3 \mathrm{c}$, despite the fact that the latter considers only a subset of ring candidates. This apparent contradiction can be explained if one considers that although solution $3 \mathrm{~b}$ leads to a better total ring mileage, it does not take into account how working lightpaths and spare wavelengths will be accommodated within the chosen set of rings.

It is interesting to notice that a lower bound on the total wavelength mileage, which would be required by the dedicated end-to-end path protection in any network, is obtained by doubling the working mileage of the SP algorithm (solution 3a). The resulting value is comparable with the total wavelength mileage required by the multi-SHR/WDM network architecture (solutions 1 and 2).

Figs. 7 and 8 report the distribution of working lightpath lengths found by both the SO-WRL solution and the SP routing, respectively. These figures confirm that, as expected, the SO-WRL solution does not yield minimum working mileage. However, the excess length of the working paths

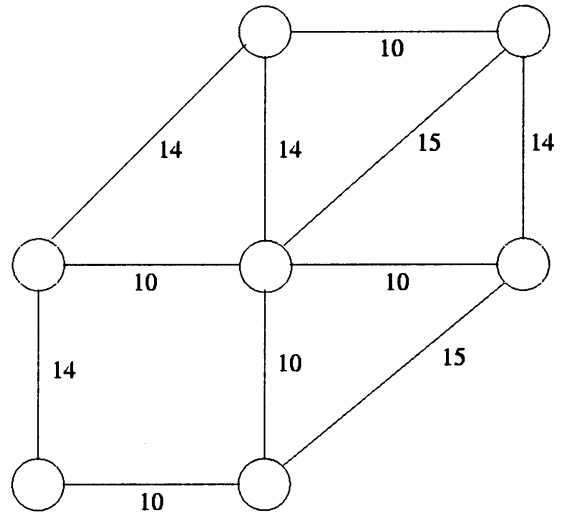

Fig. 6. Seven-node benchmark network.

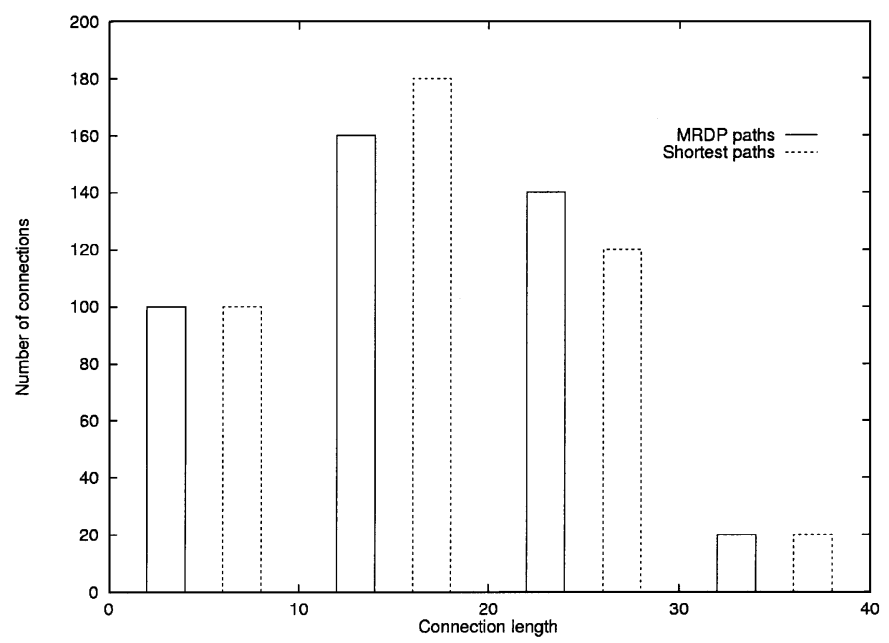

Fig. 7. Benchmark network: lightpath length distribution when all rings are used.

selected by MRDP algorithm is marginal when compared to the length of the shortest paths.

\section{B. European Network}

In this section, the SO-WRL approach is applied to the European network shown in Fig. 9 that consists of 19 nodes and 78 unidirectional lines. The traffic matrix is assumed to be nonuniform, symmetric, and complete. The total number of requested lightpaths is $1352 .{ }^{5}$ Details on the line mileage and traffic matrix used in this set of experiments can be found in [20].

When the maximum ring size equals 3 , a solution to the WRL problem cannot be found with the proposed SO-WRL approach.

${ }^{5} \mathrm{~A}$ node pair may require more than one lightpath. 


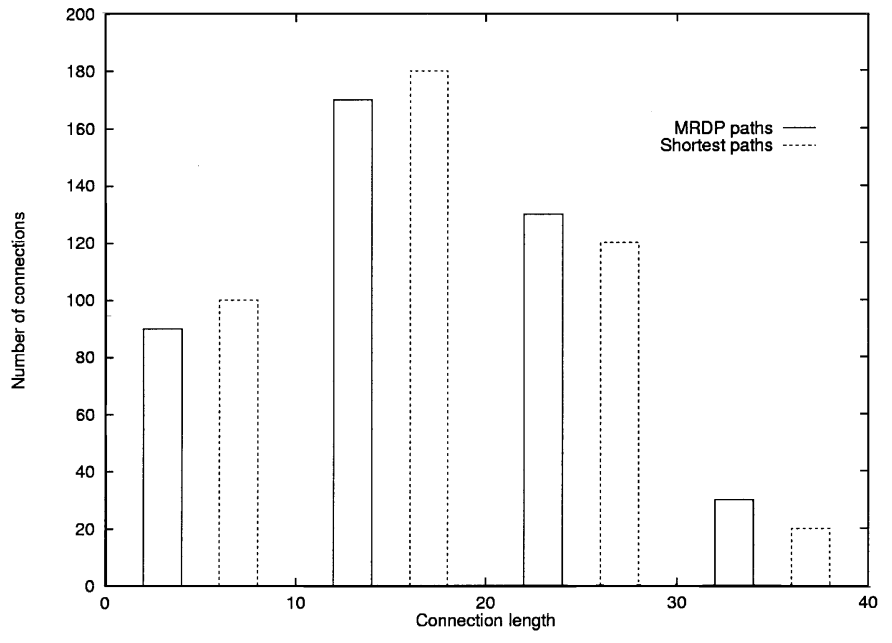

Fig. 8. Benchmark network: lightpath length distribution when shortest rings only are used.

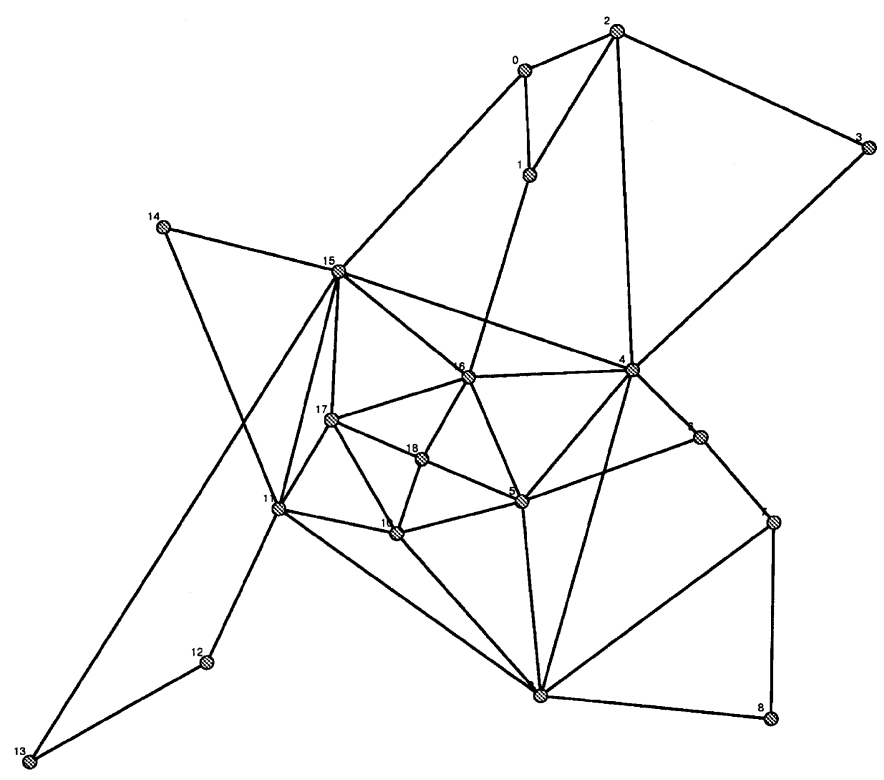

Fig. 9. European network.

All the solutions found for $N_{r}=3$ require that the candidate ring set be augmented to include some larger rings, as indicated in Section III-C1.

1) Problem Complexity Versus Wavelength Mileage: Recall that the number of path candidates in set $P_{s d}, k$ determines the size of the search space explored by the solver. The solution space is also proportional to the number of ring candidates in set $R$ that is a function of $N_{r}$. Theoretically, for any given value of $N_{r}$, the optimal solution found with $k$ is not worse than the solution found with $k^{\prime}<k$. However, due to the complexity of the problem and the size of the considered network, a tradeoff is found between the size of the search space and the optimality of the solution found by the solver in five hours of computational time on a pentium PC running at $933 \mathrm{MHz}$. For example, the curves in Fig. 10 show a nearly theoretical behavior: by increasing $N_{r}$ or/and $k$ the total wavelength mileage decreases. However, when $k \rightarrow \infty$ or when both values of $k$ and $N_{r}$ are large, the curves do not follow the theoretical behavior, because

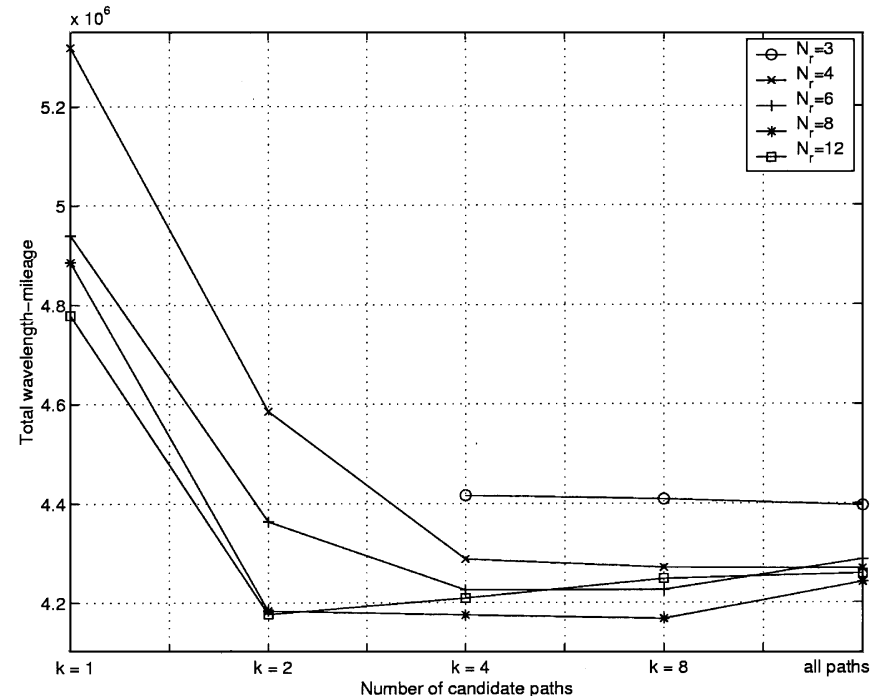

Fig. 10. European Network: total wavelength mileage under constrained design, i.e., $N_{l}=2$ and $N_{v}=4$.

the increasing complexity of the problem makes the suboptimal solutions found in five hours less close to the optimal.

The complexity of the problem is minimized when $k=1$, i.e., the working lightpaths are routed using the shortest path found by the MRDP algorithm. In this case, only the ring cover and the spare wavelengths are computed by the ILP solver. With $k=1$, the working mileage is reduced, but the overall mileage (working lightpaths and spare wavelengths) suffers considerable penalty when compared to solutions with $k>1$. Depending on $N_{r}$, this penalty may grow up to $20 \%$. These results confirm the tradeoff between the minimization of the working lightpath miles and the minimization of the provisioned spare wavelength mileage in the presence of bounded computational time.

2) Varying the Maximum Ring Size $N_{r}$ : Theoretically, larger values of $N_{r}$ must yield solutions with decreasing wavelength mileage as more ring candidates become available in the optimization process. However, from a practical point of view, the maximum ring size has a tangible effect on the total wavelength mileage only over a limited range of values. For example, in the European network the wavelength mileage reduction becomes marginal for values of $N_{r}>6$ (Figs. 10 and 11). Additional evidence of this trend is reported in Table II. For varying values of $N_{r}$ the table shows the average-obtained over different solutions with different $k$ - of the number of rings, and of the ring size in set $R$ (obtained using the SR algorithm) and in set $R_{o}$, i.e., the ring cover, with $\left(N_{l}=2\right.$ and $\left.N_{v}=4\right)$ and without $\left(N_{l}, N_{v} \rightarrow \infty\right)$ network design constraints. The average ring size in the cover does not grow proportionally with $N_{r}$, especially in the presence of design constraints. Results marked with an asterisk indicate that rings exceeding the maximum ring size are required to obtain a feasible solution (Section III-C1).

3) Effects of Design Constraints: Bounding the maximum number of rings per line and per node (constrained design) influences both the selection of the cover rings and, indirectly, the total wavelength mileage. In the unconstrained design case $\left(N_{l}, N_{v} \rightarrow \infty\right)$, the total wavelength mileage is reduced by up to $10 \%$ (Fig. 11) when compared to the constrained design 
TABLE II

European Network: Average Number of Rings and Average Ring Size ( $R$ : Set OF CANDidate Rings, $R_{o}$ : Rings In the Cover Found).

\begin{tabular}{c|c|c|c|c|c|c}
\hline$N_{r}$ & \multicolumn{3}{|c|}{ \# of rings } & \multicolumn{3}{c}{ Average \# of nodes per ring } \\
\hline & $R$ & $R_{o}$ with constr. & $R_{o}$ without constr. & $R$ & $R_{o}$ with constr. & $R_{o}$ without constr. \\
\hline $3^{*}$ & $19^{*}$ & $13.3^{*}$ & $17^{*}$ & $3.21^{*}$ & $3.3^{*}$ & $3.23^{*}$ \\
\hline 4 & 30 & 14.6 & 20.6 & 3.5 & 3.3 & 3.52 \\
\hline 6 & 63 & 11.8 & 30.2 & 4.56 & 3.42 & 4.63 \\
\hline 8 & 93 & 10.8 & 35.5 & 5.46 & 4.29 & 5.17 \\
\hline 12 & 112 & 9.8 & 37 & 6.19 & 5.1 & 5.33 \\
\hline
\end{tabular}

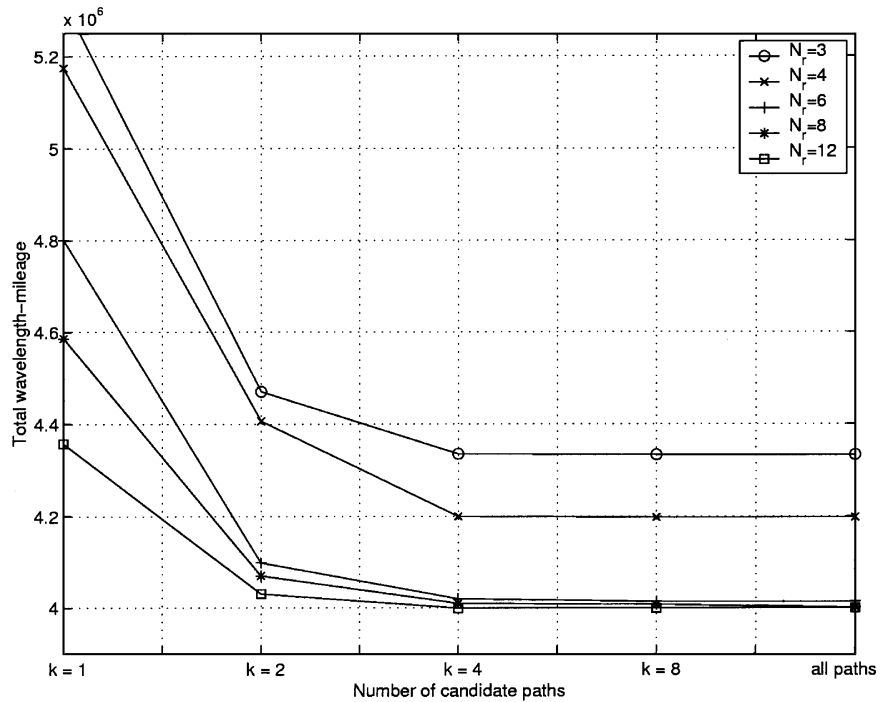

Fig. 11. European Network: total wavelength mileage under unconstrained design.

(Fig. 10). However, in the unconstrained design case, the number of required rings is up to nearly four times (Table II) when compared to the constrained case, thus, significantly complicating the network management.

If the design constraints are too stringent, the problem may not have a feasible solution even when the ring candidates in $R$ cover all the lines of the mesh. This may be the case when the limited number of path candidates in $P_{s d}$ does not allow to route the working lightpaths over the constrained ring cover (Fig. 10, cases $N_{r}=3$ and $k \leq 4$ ).

Table II illustrates the effect of the design constraints on the number of cover rings and number of nodes per ring in the solutions found for the European network. Under constrained design, the number of rings is almost the same in every solution, independently of the number of ring candidates in $R$. The average ring size in the cover increases marginally as a function of the average size of the ring candidates in set $R$. Under unconstrained design, more rings are selected to be in the cover. As $N_{r}$ increases, the average size of the rings in the cover grows more significantly than that under the constrained design.

In conclusion, when compared to the constrained design, the unconstrained design yields reduced wavelength mileage, but, on the other hand, requires more complex node hardware and network management.

4) Limited Wavelength Conversion Availability Case: The scenario in which wavelength conversion availability is limited to OXCs is analyzed in this section. The average number of wavelength converters per lightpath is plotted in Figs. 12 and

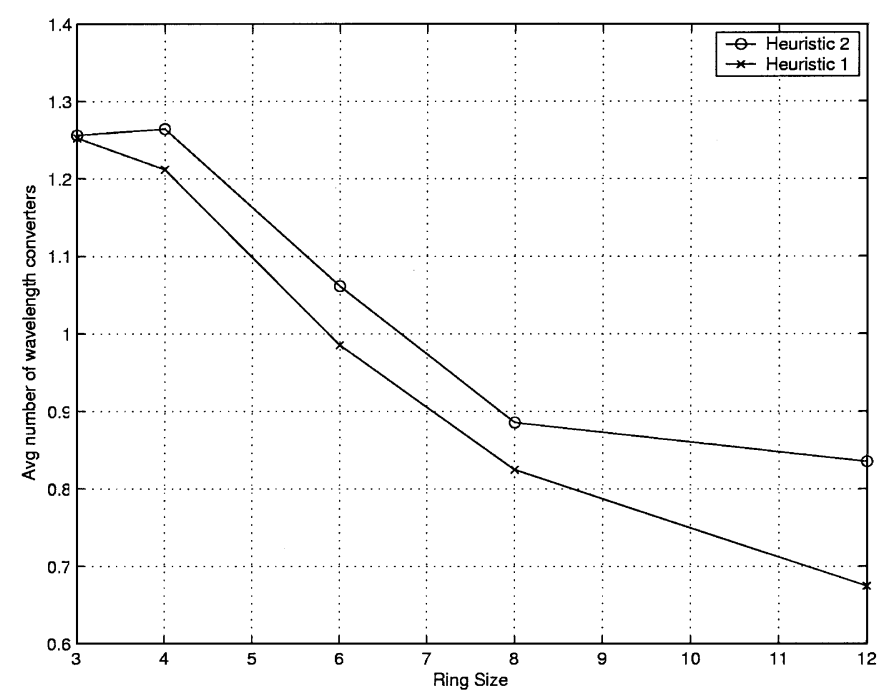

Fig. 12. Average number of wavelength converters per lightpath under constrained design, i.e., $N_{l}=2$ and $N_{v}=4$.

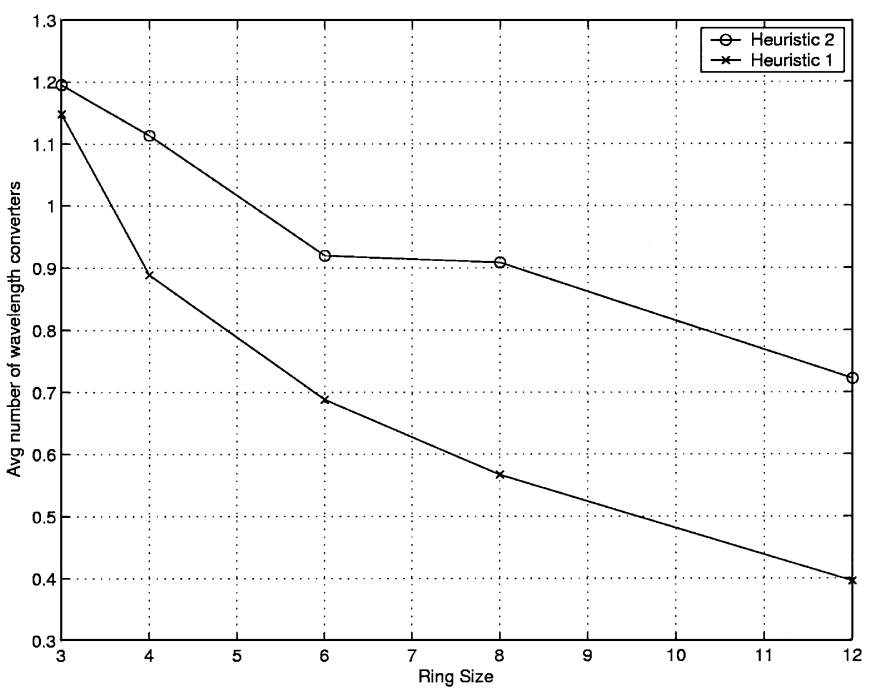

Fig. 13. Average number of wavelength converters per lightpath under unconstrained design.

13 for the constrained and unconstrained design case, respectively. The total wavelength mileage for the two cases are plotted in Figs. 14 and 15, respectively. Plots are obtained by varying the maximum ring size and using $k=8$. The plots show that as the ring size increases, the number of required wavelength converters decreases. This is due to the increasing number of lightpaths that can be accommodated using a single ring. The plots illustrate clearly the tradeoff between the two heuristics 


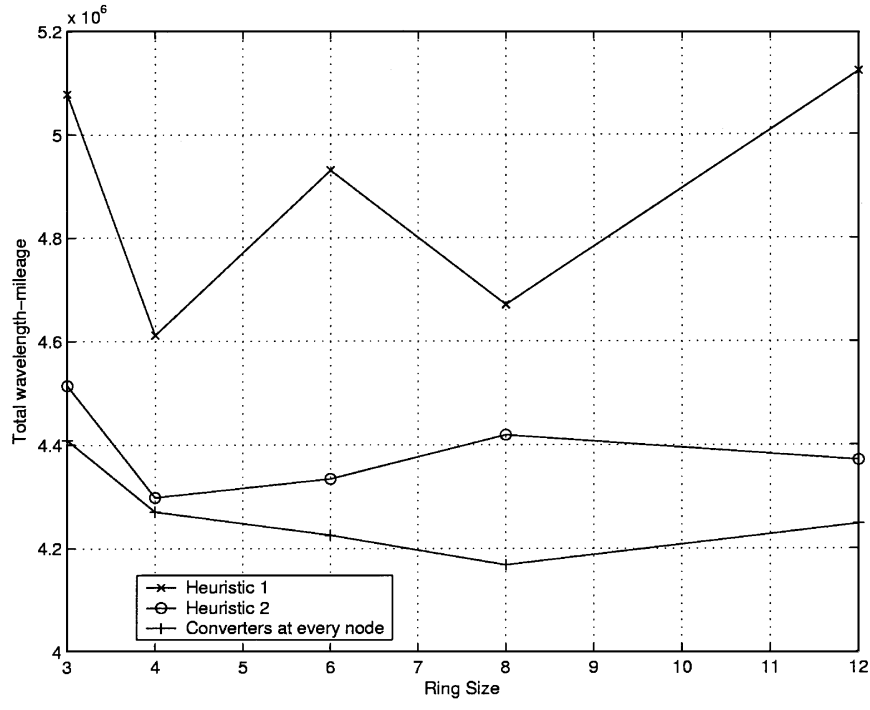

Fig. 14. Wavelength mileage under constrained design, i.e., $N_{l}=2$ and $N_{v}=4$.

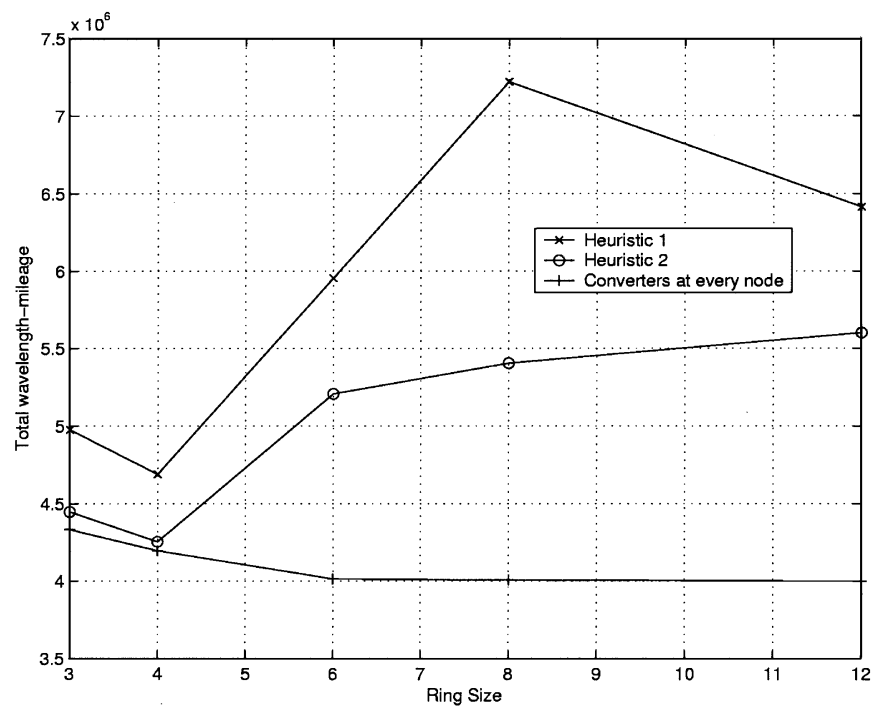

Fig. 15. Wavelength mileage under unconstrained design.

proposed in Section IV. The number of wavelength converters can be reduced at the price of an increased wavelength mileage.

\section{SUMMARY}

This paper addressed the problem of optimally designing WDM networks with arbitrary topologies using self-healing WDM rings (SHRs/WDM), referred to as the WRL problem. The proposed solutions take into account three practical design constraints: the maximum ring size, the maximum number of rings allowed to share a line, and the maximum number of rings allowed to share a node. Exact and simplified suboptimal ILP formulations of the WRL problem that yield solutions with minimal total wavelength mileage were proposed and compared. With the proposed formulations, it is possible to jointly and optimally solve the lightpath routing, the ring cover, and the spare wavelength provisioning subproblems that constitute the WRL problem. Using a benchmark network, it was demon- strated that the joint solution of the three subproblems yields a total wavelength mileage reduction that is up to $12 \%$ when compared to solutions that deal with the three subproblems sequentially. In addition, the suboptimal solution was found to reduce the complexity of the WRL problem significantly, still yielding results that are only 1\%-2\% worse than the optimum solution.

The proposed approach to designing survivable WDM networks is therefore close to optimal, and, more importantly, practical, because: 1) relatively large networks with dozens of nodes can be designed [25], 2) the worst case recovery time of the SHR/WDM can be determined by limiting the maximum size of the rings, and 3) node hardware and network management complexity can be limited by bounding the maximum number of rings that may share the same node and the same line.

Finally, two heuristics were proposed that trade wavelength mileage for a reduced number of wavelength converters. The proposed heuristics require converters at the optical crossconnects only, thus allowing converter-free optical add and drop multiplexers to be used in the rings.

\section{REFERENCES}

[1] IEEE J. Select. Areas Commun., vol. 20, Jan. 2002. Special Issue on WDM-based Network Architectures.

[2] I. Chlamtac, A. Ganz, and G. Karmi, "Lightpath communications: A novel approach to high bandwidth optical WANs," IEEE Trans. Commun., vol. 40, pp. 1171-1182, July 1992.

[3] C. A. Brackett, "Dense wavelength division networks: Principles and applications," IEEE J. Select. Areas Commun., vol. 8, pp. 948-964, June. 1990.

[4] "Architecture of Optical Transport Neworks," Int. Telecommun. Union, Telecommun. Standardization Sector (ITU-T), Geneva, Switzerland, Recommendation G.872, Feb. 1999.

[5] T. Wu, Fiber Optic Survivability. Norwood, MA: Artech House, 1992.

[6] J. Shi and J. Fonseka, "Hierarchical self-healing rings," IEEE/ACM Trans. Networking, vol. 3, pp. 690-697, Dec. 1995.

[7] T. Shiragaki, N. Henmi, T. Kato, M. Fujiwara, M. Misono, T. Shiozawa, and S. Suzuki, "Optical cross-connect system incorporated with newly developed operation and management system," IEEE J. Select. Areas Commun., vol. 16, pp. 1179-1189, Sept. 1998.

[8] J. Armitage, O. Crochat, and J. L. Boudec, "Design of a survivable WDM photonic network," in Proc. IEEE INFOCOM, vol. 1, Apr. 1997, pp. 244-252.

[9] D. Johnson, "Survivability strategies for broadband networks," in Proc. IEEE GLOBECOM'96, vol. 1, Nov. 1996, pp. 452-456.

[10] L. Berthelon, P. Gavignet, P. A. Perrier, S. Ruggeri, A. Noury, C. Coeurjolly, V. Havard, S. Gauchard, and H. Fevrier, "Toward photonic switching: Experimental demonstration of a reconfigurable survivable WDM ring nework," in Proc. IEEE GLOBECOM, vol. 1, Nov. 1996, pp. 311-315.

[11] R. Ramaswami and K. N. Sivarajan, Optical Networks: A Practical Perspective. San Mateo, CA: Morgan Kaufmann, 1998.

[12] T. Stern and K. Bala, Multiwavelength Optical Networks. A Layered Approach. Reading, MA: Addison-Wesley, May 1999.

[13] B. Mukherjee, Optical Communication Networks. New York: McGraw-Hill, 1997.

[14] C. Lee and S. Chang, "Balancing loads on SONET rings with integer demand splitting," Comput. Oper. Res., vol. 24, no. 3, pp. 221-229, 1997.

[15] J. Shi and J. Fonseka, "Interconnection of self-healing rings," in Proc IEEE Int. Communications Conf., vol. 3, June 1996, pp. 1563-1567.

[16] J. Slevinsky, W. Grover, and M. MacGregor, "An algorithm for survivable network design employing multiple self-healing rings," in Proc. IEEE GLOBECOM, vol. 3, Nov.-Dec. 1993, pp. 1568-1573.

[17] L. Nederlof, M. Garnot, and F. Masetti, "A comparison of survivability methods for the optical layer," in Proc. Network and Optical Communications, June 1997, pp. 103-110.

[18] L. Wuttisittikulkij and M. O'Mahony, "Design of a WDM network using a multiple ring approach," in Proc. IEEE GLOBECOM, vol. 1, Nov. 1997, pp. 551-555. 
[19] A. Lardies, R. Jagannathan, A. Fumagalli, I. Cerutti, and M. Tacca, "A flexible WDM ring network design and dimensioning methodology," presented at the IFIP TC6 4th Working Conf. Optical Network Design and Modeling (ONDM), Athens, Greece, Jan. 2000.

[20] I. Cerutti and M. Tacca, "Optimal self-healing ring protection and routing in WDM survivable networks," Tesi di Laurea, Politecnico di Torino, Torino, Italy, Dec. 1998.

[21] G. Ellinas, A. Hailemariam, and T. Stern, "Protection cycles in mesh WDM networks," IEEE J. Select. Areas Commun., vol. 18, pp. 1924-1937, Oct. 2000

[22] P. Mateti and N. Deo, "On algorithms for enumerating all circuits of a graph," SIAM J. Comput., vol. 5, no. 1, Mar. 1976.

[23] M. Gondran and M. Minoux, Graphs and Algorithms. New York: Wiley Interscience, 1979.

[24] M. Berkelaar, "lpsolve 2.2," Eindhoven Univ. Technol., Design Automation Section, Eindhoven, The Netherlands.

[25] A. Fumagalli, I. Cerutti, M. Tacca, D. Montgomery, I. Chlamtac, and K. Pathak, "CATO: Trans-layer dense wavelength division multiplexing (DWDM) network optimization: The clustering approach for large network design," in Proc. OSA/IEEE Optical Fiber Communication Conf., Baltimore, MD, Mar. 2000, poster presentation WN57.

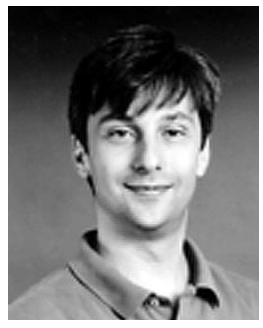

Andrea Fumagalli (M'00) received the Laurea degree in electronics engineering in 1987 and the Ph.D. degree in electrical engineering from the Politecnico di Torino, Torino, Italy, in 1992.

Since 1997, he has been an Associate Professor of electrical engineering with the University of Texas at Dallas, becoming the Head of the Optical Networking Advanced Research (OpNeAR) Laboratory in 2000. From 1992 to 1998 , he was an Assistant Professor of the electronics engineering with the Politecnico di Torino. He served as Guest Editor of a special issue on WDM networks for the European Transactions on Telecommunications. His research interests include high-speed and optical networking, survivable networks, and sensor networks.

Dr. Fumagalli served as Technical Co-Chair of the High Speed Networks Symposium of IEEE GLOBECOM 2002.

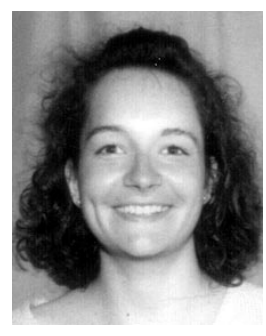

Isabella Cerutti (S'00-M'02) received the Laurea degree in electrical enginnering from the Politecnico di Torino, Torino, Italy, in 1998 and the Ph.D. in electrical engineering from the University of Texas at Dallas in 2002

She is currently a Research Associate with the University of Texas at Dallas. Her research interests include planning, optimization, performance evaluation, and fault protection and restoration of optical networks.

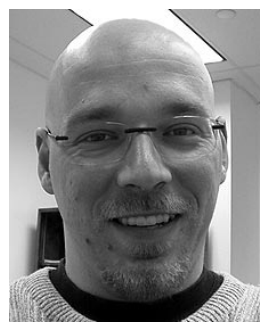

Marco Tacca (S'00-M'02) received the Laurea degree from the Politecnico di Torino, Torino, Italy, in 1998 and the Ph.D. degree from the University of Texas at Dallas in 2002.

$\mathrm{He}$ is currently a Senior Lecturer with the University of Texas at Dallas. His research interests include aspects of optical networks, high-speed photonic network planning, fault protection and restoration, and performance evaluation. 